\title{
The Properties of Determinative Disjunctive Normal Forms and Systems Based on Them
}

\author{
Anahit Chubaryan ${ }^{1}$, Armine Chubaryan ${ }^{1} \&$ Arman Tshitoyan $^{1}$ \\ ${ }^{1}$ Department of Informatics and Applied Mathematics, Yerevan State University, Armenia \\ Correspondence: Anahit Chubaryan, Department of Informatics and Applied Mathematics, Yerevan State Univer- \\ sity, 1 Alek Manoogyan str., Yerevan, Armenia. Tel: 374-9327-9118. E-mail: achubaryan@ysu.am
}

Received: September 4, 2012 Accepted: October 25, 2012 Online Published: November 26, 2012

doi:10.5539/jmr.v4n6p89 URL: http://dx.doi.org/10.5539/jmr.v4n6p89

\begin{abstract}
We study some structural and numerical properties of varieties for determinative disjunctive normal forms, introduced here for given propositional formula. We consider for classical and non-classical propositional logics some proof systems, which are constructed on the base of determinative disjunctive normal forms. We investigate also the relation between the proof complexities in some well-known classical and non-classical proof systems (Resolution, Cut-free sequent, Gentzen refutation, Cutting planes etc.) and numerical properties of varieties for determinative disjunctive normal forms for classical and non-classical tautologies.
\end{abstract}

Keywords: Boolean function, propositional formula, determinative conjunct, determinative disjunctive normal form, propositional proof system, proof complexity, polynomial simulation

\section{Introduction}

Many of the proof systems of classical propositional logic use presentation of tautologies or contradictions in disjunctive normal forms (DNF) or in conjuctive normal forms (CNF). Some of the outstanding examples of propositional tautologies (contradictions) can be presented in various DNF (CNF) and proof complexities of these presentations can differ from each other.

It is well known that a Boolean function can be represented by different $D N F$ : perfect, abridged, dead-end, shortest and minimal (see for example in Diskretnaya (1974)). It is also known that every conjunct from abridged (therefore from every dead-end, every minimal and at least one shortest) $D N F$ corresponds to some prime implicant. If we consider a tautology in the capacity of the Boolean function, then it has a unique prime implicant, which corresponds to empty conjunct, but it is well known that there are "hard" and "simple" tautologies, therefore the representations of classical tautologies, moreover of non-classical tautologies, in some above varieties of $D N F$ are not entirely correct.

Using some notions given in Chubaryan, An. and Chubaryan, Arm. (2007) for tautologies, in this paper for given propositional formula we introduce the notions of determinative conjunct, minimal determinative conjunct, to which corresponds determinative prime interval, determinative disjunctive normal form $(d D N F)$, perfect $d D N F$, abridged $d D N F$, dead-end $d D N F$, shortest $d D N F$ and minimal $d D N F$. We compare the properties of $D N F$ and $d D N F$ for the same formula. We show that there are some essential difference between the numerical and structural properties of the same varieties of $D N F$ and $d D N F$ for a given formula. Then we investigate the relations between numerical properties for the varieties of dDNF and proof complexities for classical and non-classical tautologies in the systems, based on $d D N F$, and in some other proof systems.

This paper consists of 5 sections. The notions of $\mathrm{dDNF}$ and its varieties for given propositional formula and, in particular, for classical tautologies as well as the structural and numerical properties of $d D N F$ are given in Section 2. The algorithm of constructions of $d D N F$ for non-classical tautologies are given in Section 3. The systems for classical and non-classical propositional logics, based on $d D N F$, are defined in Section 4. In the same place the relations between the numerical properties of $d D N F$ and proof complexities in described systems as well as in some other proof systems are investigated. The conclusions are given in the Section 5.

An extended abstract of some parts of this paper appeared as Chubaryan, An., Chubaryan, Arm. and Abajyan (2011). 


\section{Determinative Disjunctive Normal Forms}

\section{1 dDNF for Given Propositional Formula}

We will use the current concepts of the unit Boolean cube $\left(E^{n}\right)$, interval in it, a propositional formula, a classical tautology, a proof system for classical propositional logic, and proof complexity. The particular choice of a language for presented propositional formulas is immaterial in this consideration. However, because of some technical reasons we assume that the language contains the propositional variables $p_{i}(i \geq 1)$ and (or) $p_{i_{j}}(i \geq 1 ; j \geq 1)$, logical connectives $\neg, \&, \vee, \supset, \equiv$ and parentheses $($, ). Note that some parentheses can be omitted in generally accepted cases.

Following the usual terminology we call the variables and negated variables literals. The conjunct $K$ (clause) can be represented simply as a set of literals (no conjunct contains a variable and its negation simultaneously).

In Chubaryan and Chubaryan (2007) the following notions were introduced.

We call a replacement-rule each of the following trivial identities for a propositional formula $\psi$ :

$$
\begin{array}{llll}
0 \& \psi=0, & \psi \& 0=0, & 1 \& \psi=\psi, & \psi \& 1=\psi, \\
0 \vee \psi=\psi, & \psi \vee 0=\psi, & 1 \vee \psi=1, & \psi \vee 1=1, \\
0 \supset \psi=1, & \psi \supset 0=\bar{\psi}, & 1 \supset \psi=\psi, & \psi \supset 1=1, \\
\overline{0}=1, & \overline{1}=0, & \overline{\bar{\psi}}=\psi, & \\
0 \equiv \psi=\bar{\psi}, & \psi \equiv 0=\bar{\psi}, & 1 \equiv \psi=\psi, & \psi \equiv 1=\psi .
\end{array}
$$

Application of a replacement-rule to some word consists in the replacing of some its subwords, having the form of the left-hand side of one of the above identities, by the corresponding right-hand side.

Let $\varphi$ be a propositional formula, $P=\left\{p_{1}, p_{2}, \ldots, p_{n}\right\}$ be the set of all variables of $\varphi$, and $P^{\prime}=\left\{p_{i_{1}}, p_{i_{2}}, \ldots, p_{i_{m}}\right\}$ $(1 \leq m \leq n)$ be some subset of $P$.

Definition 1 Determinative conjunct (Chubaryan \& Chubaryan, 2007). Given $\sigma=\left\{\sigma_{1}, \ldots, \sigma_{m}\right\} \subset E^{m}$, the conjunct $K^{\sigma}=\left\{p_{i_{1}}^{\sigma_{1}}, p_{i_{2}}^{\sigma_{2}}, \ldots, p_{i_{m}}^{\sigma_{m}}\right\}$ (Note 1$)$ is called $\varphi$ - 1-determinative $\left(\varphi\right.$-0-determinative) if assigning $\sigma_{j}(1 \leq j \leq m)$ to each $p_{i_{j}}$ and successively using replacement-rules we obtain the value of $\varphi(1$ or 0$)$ independently of the values of the remaining variables.

$\varphi$-1-determinative conjunct and $\varphi$-0-determinative conjunct are called also $\varphi$-determinative or determinative for $\varphi$.

Example 1 For $\varphi=\left(p_{1} \supset\left(p_{2} \supset p_{1}\right)\right) \& p_{3}$ the conjuncts $\left\{p_{1}, p_{2}, p_{3}\right\},\left\{p_{1}, p_{3}\right\},\left\{\bar{p}_{1}, p_{3}\right\}$ are $\varphi-1$-determinative and $\left\{p_{1}, \bar{p}_{3}\right\},\left\{\bar{p}_{3}\right\}$ are $\varphi-0$-determinative, but $\left\{p_{2}, p_{3}\right\}$ isn't $\varphi$-determinative.

Definition 21 -determinative for $\varphi$ conjunct $K^{\sigma}=\left\{p_{i_{1}}^{\sigma_{1}}, p_{i_{2}}^{\sigma_{2}}, \ldots, p_{i_{m}}^{\sigma_{m}}\right\}$ is called minimal determinative if no subset of $K^{\sigma}$ is determinative for $\varphi$.

Note that minimal determinative conjunct corresponds to determinative prime interval.

In above example $\left\{p_{1}, p_{3}\right\}$ and $\left\{\bar{p}_{1}, p_{3}\right\}$ are minimal determinative, but $\left\{p_{1}, p_{2}, p_{3}\right\}$ is not minimal $\varphi$-determinative.

Definition $3 D N F \quad D=\left\{K_{1}, K_{2}, \ldots, K_{l}\right\}$ is called determinative $D N F(d D N F)$ for $\varphi$ if $\varphi=D$ and every conjunct $K_{j}(1 \leq i \leq j)$ is 1-determinative for $\varphi$.

It is obvious that for every propositional formula $\varphi$ perfect $D N F$ is $\varphi$-determinative, but not every $D N F$ for $\varphi$ is $d D N F$.

Example 2 For $\varphi=p_{1} \& p_{2} \vee \bar{p}_{2}, D=\left\{p_{1}, \bar{p}_{2}\right\}$ is $D N F$ for $\varphi$, but it isn't $\varphi$-determinative, because conjunct $\left\{p_{1}\right\}$ isn't $\varphi$-determinative.

Basing on these notions and following Diskretnaya (1974) we introduce the notions of determinative versions for above mentioned $D N F$ and search the numerical characteristics of $d D N F$.

So,

1) $d D N F$ for formula $\varphi$ is called abridged determinative $D N F(a d D N F)$ if it consists of all minimal $\varphi$-determinative conjuncts.

2) $d D N F$ for formula $\varphi$ is called dead-end determinative $D N F(d d D N F)$ if after the removal of any its conjunct it does not be equal to $\varphi$. 
3) $d D N F$ for formula $\varphi$ is called shortest determinative $D N F$ (shdDNF) if it consist of the minimal number of conjuncts among all $d D N F$ of $\varphi$.

4) $d D N F$ for formula $\varphi$ is called minimal determinative $D N F(m d D N F)$ if it has the minimal number of literals among all $d D N F$ of $\varphi$.

The abridged normal form is uniquely constructed from a Boolean function by means of a fairly simple algorithm. Its most important property is the fact that every minimal disjunctive normal form of a function and at least one shortest form can be obtained from the abridged disjunctive normal form by the elimination of certain elementary conjunctions. Therefore many minimization algorithms employ the abridged disjunctive normal form as the initial specification of a Boolean function (see for example in Diskretnaya, 1974).

The abridged $d D N F$ for every formula $\varphi$ has the same property, therefore we can use the same well known algorithms to obtain shortest and minimal $d D N F$ from abridged $d D N F$, but in the case of $d D N F$ we must verify the determinative property for each conjunct, which is obtained after every step of the algorithm. Note that determinative property of conjunct can be verified on-line.

\subsection{The Properties of dDNF for Classical Tautologies}

As only tautologies (classical or non-classical) are provable in propositional proof systems, further we will consider $d D N F$ for tautologies. Here we investigate some structural and numerical properties of various types of $d D N F$ for classical tautologies.

It is easy to prove the following

Proposition 1 Each dDNF for given tautology must have at least one occurrence of some variable with negation.

Really, otherwise $d D N F$ can not "cover" the points $(0,0, \ldots, 0)$ from unit Boolean cube.

It is well known that minimal DNF for monotone Boolean function has no occurrence of variable with negation. The situation with $d D N F$ is other.

Proposition 2 There are monotone Boolean functions each dDNF for which (therefore mdDNF) must have at least one occurrence of some variable with negation.

Such monotone Boolean functions correspond to tautologies.

Proposition 3 The maximal number of conjuncts in shdDNF for tautologies in $n$ variables is equal to $2^{n}$.

Proof of upper bound can be obtained by induction, using Proposition 1 . The lower bound follows by consideration of the tautologies

$$
\alpha_{n}=p_{1} \equiv p_{2} \equiv \ldots \equiv p_{n} \equiv p_{1} \equiv p_{2} \equiv \ldots \equiv p_{n}
$$

Note that the maximal number of conjuncts in shortest $D N F$ for Boolean functions in $n$ variables is $2^{n-1}$ (see in Diskretnaya, 1974).

Each $d D N F$ for every formula $\varphi$ is $D N F$ for Boolean function, which is presented by a formula $\varphi$, therefore the various numerical characteristics of $D N F$ are valid for $d D N F$.

If we consider only tautologies, then the upper bounds for numerical characteristics of DNF are also valid for $d D N F$. For the lower bounds we can construct the "bad" examples of tautologies on the base of the "bad" formulas from Diskretnaya (1974), using the following

Proposition 4 If the formula $\varphi$ is disjunction of the formulas $\varphi_{1}$ and $\varphi_{2}$, then $\varphi-1$-determinative conjunct must be $\varphi_{1}-1$-determinative or $\varphi_{2}-1$-determinative.

In particular, if we denote by $L^{a}(n)$ the maximal number of conjuncts in $a d D N F$ for tautologies in $n$ variables, then we can prove

Proposition $5 L^{a}(n)=O\left(\frac{3^{n}}{\sqrt{n}}\right)$ and $L^{a}(n)=\Omega\left(\frac{3^{n}}{n}\right)$.

Proof of upper bound is the same as in Diskretnaya (1974). For proving the lower bound we must "complete until sequence of tautologies" the sequence, considered in Diskretnaya (1974) "bad" formulas, by adding to each formula two formulas with disjunction.

So let $S_{n}^{[i, k]}(0 \leq i \leq k \leq n)$ be Boolean function in $n$ variables such that 


$$
\forall\left(\sigma_{1} \ldots \sigma_{n}\right) \in E^{n} \quad S_{n}^{[i, k]}\left(\sigma_{1} \ldots \sigma_{n}\right)=1 \text { iff } i \leq \sum_{i=1}^{n} \sigma_{i} \leq k
$$

and by $D^{a}\left(S_{n}^{[i, k]}\right)$ is denoted the abridged $D N F$ of $S_{n}^{[i, k]}$. As sequence of "bad" tautologies we consider the following formulas for $n \geq 3$.

$$
\varphi_{n}=D^{a}\left(S_{n}^{[0,[n / 3]-1]}\right) V D^{a}\left(S_{n}^{[[n / 3], 2[n / 3]]}\right) V D^{a}\left(S_{n}^{[2[n / 3]+1, n]}\right) .
$$

It is not difficult to see that abridged $D N F$, therefore $d D N F$ of $\varphi_{n}$ has

$$
C_{n}^{[n / 3]-1}+C_{n}^{[n / 3]} * C_{n-[n / 3]}^{[n / 3]}+C_{n}^{2[n / 3]+1}
$$

various conjuncts, each of which is $\varphi_{n}$-determinative.

Note that the sequence of Boolean functions $S_{n}^{[n / 3], 2[n / 3]}$ is considered in Diskretnaya (1974).

\section{Determinative Disjunctive Normal Forms for Non-classical Tautologies}

The investigations of proof complexity start for the systems of Classical Propositional Logic (CPL). However, natural real conclusions have constructive character in most cases, therefore the investigation of the proofs complexities is important for systems of Intuitionistic Propositional Logic (IPL) and in some cases also for Minimal (Johansson's) Propositional Logic (MPL) (see for example in Kleene, 1952). In particular, logic programming is based on intuitionistic logic.

As the intuitionistic (minimal) validity is determined by derivability in some intuitionistic (minimal) propositional proof calculus, the above definition of $d D N F$ for classical tautologies is not applicable here.

Some algorithm for construction of $d D N F$ of a classical tautology $\varphi$ on the base of resolution refutation for $\bar{\varphi}$ is described in Chubaryan (2002). Following Chubaryan, An., Chubaryan, Arm. and Sayadyan (2007), we give here the corresponding algorithm on the base of intuitionistic (minimal) resolution systems.

Let us recall the mentioned systems.

The system RI (resolution for IPL) is described by Mints in Mints (1985).

The axioms are the sequents

$$
p \rightarrow p \text { and } \perp \rightarrow p
$$

The rules of inference (resolution rules) are:

$$
\begin{aligned}
& \left\{\begin{array}{l}
\frac{(p \supset q) \rightarrow r ; \Sigma, p \rightarrow \perp}{\Sigma \rightarrow r}(1) \\
\frac{(p \supset q) \rightarrow r ; \Sigma, p \rightarrow q}{\Sigma \rightarrow r} \\
\frac{(p \supset q) \rightarrow r ; \Sigma \rightarrow \perp}{\Sigma \rightarrow r} \\
\frac{(p \supset \perp) \rightarrow r ; \Sigma, p \rightarrow \perp}{\Sigma \rightarrow r}
\end{array}\right\} \\
& \frac{p \rightarrow q \vee r ; \Gamma \rightarrow p ; \Sigma q \rightarrow s^{*} ; \Pi, r \rightarrow s^{* *}}{\Gamma, \Sigma, \Pi \rightarrow s}(\vee \\
& \frac{p, q \rightarrow r^{*} ; \Gamma \rightarrow p ; \Sigma \rightarrow q}{\Gamma, \Sigma \rightarrow r^{*}}(\mathrm{cut}) \frac{p \rightarrow q ; \Gamma \rightarrow p}{\Gamma \rightarrow q}
\end{aligned}
$$

(2) $\frac{\rightarrow \perp}{\rightarrow p}(\perp)$, where $p^{*}$ for some propositional variable $p$ can be $p$ or $\perp$.

The corresponding system for MPL is defined in the following way: $R M$ is obtained from $R I$ by dropping the rules (1) and (2) (see in Chubaryan, An., Chubaryan, Arm., \& Sayadyan, 2007).

It is necessary to make some comments about the system $R I(R M)$. Let $\varphi$ be some formula and $\left\{p_{1}, p_{2}, \ldots, p_{n}\right\}$ is the set of its all variables (later we call this variables the main variables). Associating a new variable with 
every non-elementary subformula of $\varphi$, we can construct the system of disjuncts by employing method described in Mints (1985). The disjuncts of this system can be represented as the following sequents

$$
p \rightarrow q \vee r ;\left(p \supset q^{*}\right) \rightarrow r ; q_{1}, q_{2}, \ldots, q_{k} \rightarrow r^{*}
$$

Let $s$ be the variable, associated with $\varphi$ itself. Mints has shown, that the sequent $\rightarrow \quad s$ is proved in $R I$ from the axioms and from above set $(\diamond)$ of disjuncts, constructed for $\varphi$, iff the sequent $\rightarrow \varphi$ is proved in the system $N I$ (natural system for IPL (see in Mints, 1985). The same result can be proved for the systems $R M$ and $N M$ (corresponding natural system for MPL, which is obtained from $N I$ by dropping the rule $\frac{\Gamma \rightarrow \perp}{\Gamma \rightarrow A}$ ). Later for every formula $\varphi$ each of disjuncts of the set $(\diamond)$ is called the additional axiom. The axiom (additional or not) is called the main axiom if it contains at least one main variable.

Recall that there is a well-known notion of positive and negative occurrences of subformulas (or variables) in the formula or in the sequent (see for example in Chubaryan, 2002). If a variable $p$ has negative occurrence in some subformula, which in its turn has negative occurrence in the formula, we say that the variable $p$ has double negative occurrence in this formula.

It is not difficult to see, that occurrence of any variable in axioms (additional or not) or in inference rules of system $R I$ is either positive, negative or double negative, and since $\overline{\bar{p}} \sim p$ is not derivable in IPL (MPL), then not only variable or variable with negation, but also variable with double negation can serve as literal for $\varphi$-determinative conjunction in IPL.

It is natural that for any variable $p$ not only the literals $p$ and $\bar{p}$ are contrary, but $\bar{p}$ and $\overline{\bar{p}}$ also. It is not difficult to see that just the contrary pairs of literals of type $p, \bar{p}$ and $\bar{p}, \overline{\bar{p}}$ are subjected to resolution by application of inference rules of $R I$.

It is necessary to note that any $\varphi$-determinative conjunct can not include contrary literals.

The analogies of the $\varphi$-determinative d.n.f. for IPL and MPL $(\varphi-I$-determinative d.n.f. and $\varphi-M$-determinative d.n.f. accordingly) can be constructed using the following algorithm.

Let $W$ be the proof of $\rightarrow s$ in $R I(R M)$. The steps for the construction of the $\varphi-I$-determinative $(\varphi-M$ determinative) $D N F$ are the followings:

1) We transform the proof $W$ into tree-like proof $W^{\text {tree }}$ of $\rightarrow s$. Let $k$ be the number of the paths of this tree.

2) For every path $i(1 \leq i \leq k)$ between two vertices, one associated with the main axiom and another with $\rightarrow s$, we construct the conjunct $K_{i}^{\prime}$ as the set of all main variables (or their negations, or double negations), which have positive (negative or double negative) occurrence in the sequents of this path.

Then we take the conjunct $\tilde{K}_{i}$, which consists of negations of all literals of $K_{i}$ and after substitution of $\overline{\bar{p}}$ type literals with $\bar{p}$, we obtain the determinative conjunct $K_{i}$.

The $D N F D=\left\{K_{i_{1}}, K_{i_{2}}, \ldots, K_{i_{t}}\right\}(t \leq k)$, consisting of all distinct above constructed not contradictory conjuncts, is called $\varphi-I$-determinative for $R I$-proof ( $\varphi-M$-determinative for $R M$-proof).

Note that only variables with one or double negations are the literals in $I$-determinative conjuncts ( $p \supset \perp$ and $(p \supset \perp) \supset \perp$ type literals in $M$-determinative conjuncts).

Using various $R I$-proofs ( $R M$-proofs) we can construct different $\varphi-I$ - determinative $D N F(\varphi-M$ - determinative $D N F$ ) and hence we can define the varieties of $D N F$ as above for classical tautologies.

Taking into consideration that for every classical tautology $\varphi$ the formula $\neg \neg \varphi(((\varphi \supset \perp) \supset \perp))$ is intuitionistic (minimal) tautology, all above results from section 2.2 are valid for IPL (MPL).

\section{The Proof Systems, Based on Determinative Disjunctive Normal Forms}

\subsection{The Systems EC, EI and EM}

Earlier some classical propositional proof system EC is introduced by the first co-author in Chubaryan (2002).

The axioms of $E C$ aren't fixed, but for every formula $\varphi$ each conjunct from some $d D N F$ of $\varphi$ can be considered as an axiom.

The classical elimination rule $\left(C_{\varepsilon}\right.$-rule) infers $K^{\prime} \cup K^{\prime \prime}$ from clauses $K^{\prime} \cup\{p\}$ and $K^{\prime} \cup\left\{\bar{p}\right.$, where $K^{\prime}$ and $K^{\prime \prime}$ are clauses and $p$ is a variable. 
The proof in $E C$ is a finite sequence of clauses such that every clause in the sequence is one of the axioms of $E C$, or is inferred from earlier clauses in the sequence by $C_{\varepsilon}$-rule.

DNF $D=\left\{K_{1}, K_{2}, \ldots, K_{l}\right\}$ is called full (tautology) if using $C_{\varepsilon}$-rule the empty conjunction $(\emptyset)$ can be proved from the axioms $\left\{K_{1}, K_{2}, \ldots, K_{l}\right\}$.

By analogy the corresponding proof system $E I(E M)$ can be constructed for IPL (MPL).

As axiom is considered every $I$-determinative ( $M$-determinative) conjunct from some $I$-determinative $(M$ - determinative) $D N F$.

For $E I(E M)$ we take the following inference rule

$$
\begin{aligned}
& \frac{K^{\prime} \cup \bar{p} \quad K^{\prime \prime} \cup \bar{p}}{K^{\prime} \cup K^{\prime \prime}} \quad I_{\epsilon}-\text { rule } \\
& \left(\frac{K^{\prime} \cup(p \supset \perp) \supset \perp \quad K^{\prime \prime} \cup(p \supset \perp)}{K^{\prime} \cup K^{\prime \prime}} \quad M_{\epsilon}-\text { rule }\right),
\end{aligned}
$$

where $K^{\prime}$ and $K^{\prime \prime}$ are clauses and $p$ is a variable.

\subsection{Proof Complexity, Polynomial Simulation}

To indicate the role of above mentioned systems and investigate the relation between the proof complexity and numerical properties of $d D N F$, let us recall some notions and notations.

By $|\varphi|$ we denote the size of a formula $\varphi$, defined as the number of all variable entries. It is obvious that the full length of a formula, which is understood to be the number of all symbols or the number of all entries of logical signs, is bounded by some linear function in $|\varphi|$.

In the theory of proof complexity two main characteristics of the proof are: $t$-complexity, defined as the number of proof steps, and $l$ - complexity, defined as total number of proof symbols. Let $\boldsymbol{\Phi}$ be a proof system and $\varphi$ be a tautology. We denote by $t_{\varphi}^{\mathbf{\Phi}}\left(l_{\varphi}^{\mathbf{\Phi}}\right)$ the minimal possible value of $t-$ complexity $(l-$ complexity) for all the proofs of tautology $\varphi$ in $\boldsymbol{\Phi}$.

Let $\boldsymbol{\Phi}_{1}$ and $\boldsymbol{\Phi}_{2}$ be two different proof systems. Following Cook and Reckhow (1979) we recall.

Definition $4 \boldsymbol{\Phi}_{2} p-t$-simulates ( $p-l$-simulates) $\boldsymbol{\Phi}_{1}$ if there exists a polynomial $\mathrm{p}()$ such that for every formula $\varphi$ derivable both in $\boldsymbol{\Phi}_{1}$ and $\boldsymbol{\Phi}_{2} t_{\varphi}^{\boldsymbol{\Phi}_{2}} \leq p\left(t_{\varphi}^{\boldsymbol{\Phi}_{1}}\right)\left(l_{\varphi}^{\boldsymbol{\Phi}_{2}} \leq p\left(l_{\varphi}^{\boldsymbol{\Phi}_{1}}\right)\right)$.

Definition 5 The systems $\boldsymbol{\Phi}_{1}$ and $\boldsymbol{\Phi}_{2}$ are $p-t$-equivalent ( $p$-l-equivalent) iff $\boldsymbol{\Phi}_{1} p-t$-simulates $(p-l$-simulates) $\boldsymbol{\Phi}_{2}$ and $\boldsymbol{\Phi}_{2} p-t$-simulates $\left(p-l\right.$-simulates) $\boldsymbol{\Phi}_{1}$.

For CPL it is proved in Chubaryan (2002) that the systems EC, Cut-free sequent and Resolution are $p$-equivalent, in Arai (2000) it is proved that Resolution system and Gentzen refutation systems are $p$-equivalent also. For IPL (MPL) it is proved in Chubaryan, An., Chubaryan, Arm. and Sayadyan (2007) that the systems EI, RI and Multi-succedent cut-free sequent ( $E M, R M$ and Multi-succedent cut-free sequent) are $p$-equivalent. We suppose also that the analogies of "Cutting planes" and "Gentzen refutation" for IPL (MPL) can be constructed on the base of $I$-determinative ( $M$-determinative) $D N F$. So the investigation of proof complexities in $E C, E I$ and $E M$ is very important. It is interesting also which is the relation between the proof complexities in E-type systems and numerical properties of $d D N F$.

The search of this questions is in progress, but at present we have some results.

By $L(\mathcal{D}(\varphi))$ we denote the number of conjuncts in $D N F \mathcal{D}$ for formula $\varphi$.

Let $d(\varphi)$ be the number of literals in minimal determinative conjunct of $\varphi$.

Theorem 1 1) Given tautology $\varphi$

a)

b)

$$
t_{\varphi}^{E C} \leqslant 2^{|\varphi|}
$$

$$
t_{\varphi}^{E C} \geqslant L(\operatorname{shdDNF}(\varphi)) \geqslant 2^{d(\varphi)}
$$

c)

$$
l_{\varphi}^{E C} \leqslant|\operatorname{mdDNF}(\varphi)|
$$


d)

$$
l_{\varphi}^{E C} \geqslant d(\varphi) * L(\operatorname{shdDNF}(\varphi)) \geqslant d(\varphi) * 2^{d(\varphi)}
$$

2) There is a sequence of tautologies $\gamma_{n}$ such that

$$
\begin{gathered}
\left|\gamma_{n}\right|=\theta(n) \\
t_{\gamma_{n}}^{E} C=\theta\left(2^{n}\right) .
\end{gathered}
$$

The statements of the point 1 are proved very easy. Really

a) as we note above for every propositional formula $\varphi$ perfect $D N F$ is $\varphi$-determinative,

b) every $\varphi$-determinative $D N F$ must contain at least $2^{d(\varphi)}$ conjuncts, each of them must use as axiom in $E C$ proof of $\varphi$ in $E C$,

c) the sum of sizes of axioms in the proof of $\varphi$ in $E C$ can not be smaller than the size of $m d D N F$ for $\varphi$,

d) follows from $b$ ).

The proof of statement 2 is given in Chubaryan (2002) on the base of formulas

$$
\beta_{n}=T T M_{n, 2^{n-1}},
$$

where

$$
T T M_{n, m}=\bigvee_{\left(\sigma_{1}, \ldots, \sigma_{n}\right) \in E^{n}} \stackrel{m}{m}=1_{i=1}^{n} \bigvee_{i=1}^{\sigma_{j}} p_{i j} \quad\left(n \geq 1,1 \leq m \leq 2^{n}-1\right) .
$$

It is obvious that the analogous results can be proved for IPL and MPL.

\section{Conclusion}

As some proof systems for propositional logics are based on $D N F$ and every "valid statement" can admit many varieties of $D N F$, the investigation of various representation of formulas (tautologies) in $D N F$ can be very important for evaluation of proof complexities in many proof systems. In particular, if for given tautology $d(\varphi)=\Theta(|\varphi|)$, then the exponential lower bound for proof complexity of $\varphi$ in many proof systems can be obtained very easy. The relation between the numerical properties of $d D N F$ and proof complexities can be useful for "good" $D N F$ construction also.

\section{Acknowledgements}

This paper is supported by grant \#11-1b023 of SSC of Goverment of RA.

\section{References}

Arai, N. (2000). Tractability of Cut-free Gentzen-type propositional calculus with permutation inference. Theoretical Computer Science, 170, 129-144. Retrieved from http://www.nii.ac.jp/staff/detail/Arai_Noriko.shtml

Chubaryan, An. (2002). Relative efficiency of proof systems in classical propositional logic. Izv. NAN Armenii Mat., 37(5), 71-84.

Chubaryan, An., \& Chubaryan, Arm. (2007). A new conception of Equality of Tautologies. L\&PS, V(1), 3-8, Triest, Italy. Retrieved from http://www.2.units.it/.../L\&PS_Vol5No1/Chubaryan_L\&PS

Chubaryan, An., Chubaryan, Arm., \& Abajyan, A. (2011). On Determinative Disjunctive Normal Forms. Proceedings of CSIT Conference, 39-41, Yerevan, Armenia.

Chubaryan, An., Chubaryan, Arm., \& Sayadyan, S. (2007). Relative efficiency propositional proof systems for Classical and Nonclassical Propositional Logic. Perspectives on Universal Logic 265-275 Polimetrica, ISP, Monza, Italy. Retrieved from http://www.polimetrica.com.BOOKCATALOG>purescience

Cook, S., \& Reckhow, R. (1979). The relative efficiency of propositional proofs systems. Journal of Symbolic Logic, 44, 36-50. http://dx.doi.org/10.2307/2273702

Diskretnaya matematika i matematicheskie voprosi kibernetiki. (1974). Izd. Nauka, 1, Moskwa (in Russian).

Kleene, S. (1952). Introduction to Metamathematics. New York: D. Van Nostrand Company, INC. Retrieved from http://www.amazon.com/Introduction-Meta 
Mints, G. (1985). Resolution systems for nonclassical logic. Semiotika i informatika, 25, 120-133 (in Russian).

Notes

Note 1. As usual, given a propositional variable $p$ and $\sigma \in E^{1}$, by $p^{\sigma}$ we denote the function $p^{\sigma}= \begin{cases}p, & \text { if } \sigma=1, \\ \bar{p}, & \text { if } \sigma=0 .\end{cases}$ 